I. S. Borisov, E. I. Shefer, Asymptotic behavior of the mean sojourn time for a random walk to be in a domain of large deviations, Mat. Tr., 2019, Volume 22, Number 2, 3-20

DOI: https://doi.org/10.33048/mattrudy.2019.22.201

Use of the all-Russian mathematical portal Math-Net.Ru implies that you have read and agreed to these terms of use

http: //www. mathnet.ru/eng/agreement

Download details:

IP : 34.229 .45 .116

April 26, 2023, 03:11:51 


\title{
АСИМПТОТИКА СРЕДНЕГО ВРЕМЕНИ ПРЕБЫВАНИЯ СЛУЧАЙНОГО БЛУЖДАНИЯ В ОБЛАСТИ БОЛЬШИХ УКЛОНЕНИЙ
}

\author{
И. С. Борисов, Е. И. Шефер
}

В статье исследована асимптотика математического ожидания времени нахождения траектории случайного блуждания до момента времени $n$ выше удаляющейся криволинейной границы в области больших уклонений в случае, когда распределение скачка удовлетворяет условию Крамера.

Ключевые слова и фразы: случайное блуждание, среднее время пребывания в области, большие уклонения.

\section{§1. Введение и формулировка результата}

Пусть $\xi_{1}, \xi_{2}, \ldots$ - независимые одинаково распределенные случайные величины с нулевым математическим ожиданием и конечной дисперсией $\sigma^{2}:=\mathbb{E} \xi_{1}^{2}>0$. Обозначим

$$
S_{k}:=\sum_{i=1}^{k} \xi_{i}, \quad k=1,2, \ldots, n .
$$

Временем пребывания траектории $\left\{S_{k} ; k=1, \ldots, n\right\}$ выше переменного уровня $x g(\cdot)$ назовем случайную величину

$$
\tau_{n}(x g):=\sum_{k=1}^{n} I\left\{S_{k}>x g(k / n)\right\},
$$

где $I(\cdot)$ - индикатор события, $x \equiv x(n) \rightarrow \infty$ при $n \rightarrow \infty$ задает скорость удаления границы, а ограниченная положительная функция $g(t)$, $t \in(0,1],-$ конфигурацию границы в зависимости от времени.

Цель работы - исследовать асимптотическое поведение при $n \rightarrow \infty$ математического ожидания

$$
\mathbb{E} \tau_{n}(x g)=\sum_{k=1}^{n} \mathbb{P}\left\{S_{k}>x g(k / n)\right\} .
$$

Работа выполнена при частичной поддержке РФФИ (код проекта 18-01-00074).

(C) И. С. Борисов, Е. И. Шефер; 2019 
Введем в рассмотрение функцию

$$
G(t):=g^{2}(t) / t, \quad t \in(0,1] .
$$

Для формулировки нижеследующих условий нам будет удобно доопределить $G(t)$ в правой полуокрестности точки $t=1$ как $G(t):=g^{2}(1) / t$. Предположим, что существует точка $t_{0} \in(0,1]$, в которой достигается минимум функции $G(\cdot)$ и выполнены следующие условия:

1) существует такое $0<r \leq g\left(t_{0}\right)$, что

$$
G(t) \geq \max \left\{c_{0}(t), \frac{r^{2}}{t}\right\} \text { при всех } t \in(0,1]
$$

где положительная функция $c_{0}(t)$ монотонно убывает на $\left(0, t_{0}\right)$, монотонно возрастает на $\left(t_{0}, 1\right]$ и $c_{0}\left(t_{0}\right)=G\left(t_{0}\right)$;

2 ) функция $G(t)$ непрерывно дифференцируема $m_{1}$ раз в левой полуокрестности точки $t_{0}$ и $m_{2}$ раз в правой полуокрестности этой точки; при этом $m_{1}$ и $m_{2}-$ порядки соответственно первых ненулевых левой и правой производных функции $G(t)$ в точке $t=t_{0}$;

3) $x / \sqrt{n} \rightarrow \infty$ и $x / n^{1-\gamma_{m}} \rightarrow 0$ при $n \rightarrow \infty$, где

$$
\gamma_{m}:=\frac{1-1 / m}{3-2 / m}, \quad m:=\max \left\{m_{1}, m_{2}\right\}
$$

4) $\mathbb{E} e^{\lambda\left|\xi_{1}\right|}<\infty$ для некоторого $\lambda>0$.

Теорема 1. При выполнении условий 1-4 и $n \rightarrow \infty$

$$
\begin{aligned}
\mathbb{E} \tau_{n}(x g) \sim M & \left(G, t_{0}, m_{1}, m_{2}\right) \frac{n(2 m !)^{1 / m} \sqrt{t_{0}}}{\sqrt{2 \pi} g\left(t_{0}\right)}\left(\frac{\sigma}{\beta_{n}}\right)^{1+2 / m} \Gamma\left(\frac{m+1}{m}\right) \\
& \times \exp \left\{-n t_{0} \Lambda\left(\frac{x g\left(t_{0}\right)}{n t_{0}}\right)\right\},
\end{aligned}
$$

где

$$
\beta_{n}:=\frac{x}{\sqrt{n}}, \quad \Gamma(z):=\int_{0}^{\infty} y^{z-1} e^{-y} d y, \quad z>0,
$$

$\Lambda(z)$ - функция уклонений (функция Крамера) случайной величины $\xi_{1}$, T. e.

$$
\Lambda(z):=\sup _{t}\left\{t z-\log \psi_{\xi_{1}}(t)\right\}, \quad \text { где } \psi_{\xi_{1}}(t):=\mathbb{E} e^{t \xi_{1}},
$$

(здесь $\log (\infty)=\infty$ по определению), 


$$
\begin{aligned}
& M\left(G, t_{0}, m_{1}, m_{2}\right) \\
& :=\left\{\begin{array}{lll}
\frac{1}{\left|G^{\left(m_{1}\right)}\left(t_{0}-0\right)\right|^{1 / m_{1}}}, & \text { если } t_{0} \in(0,1), m_{1}>m_{2}, \\
\frac{1}{\left|G^{\left(m_{2}\right)}\left(t_{0}+0\right)\right|^{1 / m_{2}}}, & \text { если } t_{0} \in(0,1), m_{1}<m_{2}, \\
\frac{1}{\left|G^{\left(m_{1}\right)}\left(t_{0}-0\right)\right|^{1 / m_{1}}}+\frac{1}{\left|G^{\left(m_{2}\right)}\left(t_{0}+0\right)\right|^{1 / m_{2}}}, & \text { если } t_{0} \in(0,1), \quad m_{1}=m_{2}, \\
\frac{1}{\left|G^{\left(m_{1}\right)}(1-0)\right|^{1 / m_{1}}}, & \text { если } t_{0}=1 .
\end{array}\right.
\end{aligned}
$$

При $m \geq 2$ аргумент экспоненты в (1) может быть заменен следующим выражением:

$$
-\frac{\beta_{n}^{2} g^{2}\left(t_{0}\right)}{2 t_{0} \sigma^{2}}+\frac{\mathbb{E} \xi_{1}^{3} \beta_{n}^{3} g^{3}\left(t_{0}\right)}{6 t_{0}^{2} \sigma^{4} \sqrt{n}}
$$

Замечание 1. В случае $m=1$ теорема 1 доказана в [1]. Кроме того, частный случай теоремы 1 при $g(\cdot) \equiv 1$, который автоматически вкладывается в случай $t_{0}=1$ и $m=1$, содержится в [4]. Стоит также отметить, что теорема 1 допускает обобщение на случай конечного набора точек $\left\{t_{k}\right\}$, в окрестностях которых выполнены условия 1-4 со своими параметрами $m_{1}$ и $m_{2}$ для каждой точки $t_{k}$. Правая часть итоговой асимптотики в этом случае будет представлена в виде суммы правых частей в (1), вычисленных для каждой точки $t_{k}$ отдельно.

Замечание 2. В соотношении (2) в силу условия 3 второе слагаемое имеет порядок $O\left(n^{-1 / 2} \beta_{n}^{3}\right)=o\left(\beta_{n}^{2}\right)$, причем эта величина в указанной в условии 3 зоне уклонений может стремиться как к нулю, так и к бесконечности.

В теореме 1 рассматривался случай, когда функция $g(t)$ касалась повернутой параболы в одной точке (или в конечном числе точек) сегмента $(0,1]$. Следующая теорема рассматривает случай, когда на невырожденном отрезке функция $g(t)$ будет совпадать с повернутой параболой, или, другими словами, функция $G(t)$ тождественно равна своему минимальному значению на некотором невырожденном отрезке $\left[t_{1}, t_{2}\right] \subset(0,1]$.

Мы будем предполагать выполненными следующие условия, аналогичные условиям 1 и 2 теоремы 1 :

$1^{\prime}$ ) существует такое $0<r \leq g\left(t_{1}\right)$, что

$$
G(t) \geq \max \left\{c_{0}(t), \frac{r^{2}}{t}\right\} \text { при всех } t \in(0,1],
$$


где положительная функция $c_{0}(t)$ монотонно убывает на $\left[0, t_{1}\right)$, монотонно возрастает на $\left(t_{2}, 1\right]$ и $c_{0}(t)=G\left(t_{1}\right)$ при всех $t \in\left[t_{1}, t_{2}\right]$;

$\left.2^{\prime}\right)$ функция $G(t)$ непрерывно дифференцируема $m_{1}$ раз в левой полуокрестности точки $t_{1}$ и $m_{2}$ раз в правой полуокрестности точки $t_{2}$; при этом $m_{1}$ и $m_{2}$ - порядки первых ненулевых левой и правой производных функции $G(t)$ в точках $t_{1}$ и $t_{2}$ соответственно.

Для формулировки теоремы 2 введем следующее обозначение:

$$
\Lambda_{0}(z):=\Lambda(z)-z^{2} / 2 \sigma^{2}
$$

Отметим, что $z^{2} / 2 \sigma^{2}$ является первым членом ряда Крамера, т. е. первым ненулевым членом разложения функции уклонений $\Lambda(z)$ в ряд Тейлора в окрестности нуля.

Теорема 2. Пусть выполнены условия $1^{\prime}, 2^{\prime}, 3$ и 4 . Тогда при $n \rightarrow \infty$

$$
\mathbb{E} \tau_{n}(x g) \sim \frac{n^{3 / 2} \sigma \sqrt{t_{1}}}{\sqrt{2 \pi} x g\left(t_{1}\right)} J_{n}\left(t_{1}, t_{2}\right) \exp \left\{-\frac{x^{2} g^{2}\left(t_{1}\right)}{2 n t_{1} \sigma^{2}}\right\}
$$

где

В случае $m \geq 2$

$$
J_{n}\left(t_{1}, t_{2}\right):=\int_{t_{1}}^{t_{2}} \exp \left\{-n t \Lambda_{0}\left(\frac{x g\left(t_{1}\right)}{n \sqrt{t_{1} t}}\right)\right\} d t .
$$

в частности,

$$
J_{n}\left(t_{1}, t_{2}\right) \sim \int_{t_{1}}^{t_{2}} \exp \left\{\frac{g^{3}\left(t_{1}\right) x^{3} \mathbb{E} \xi_{1}^{3}}{6 \sigma^{4} t_{1}^{3 / 2} n^{2} \sqrt{t}}\right\} d t
$$

$$
J_{n}\left(t_{1}, t_{2}\right) \sim \begin{cases}t_{2}-t_{1}, & \text { если } \mathbb{E} \xi_{1}^{3}=0 \text { или } \frac{x^{3}}{n^{2}} \rightarrow 0, \\ \frac{12 n^{2} t_{1}^{3} \sigma^{4}}{x^{3} g^{3}\left(t_{1}\right) \mathbb{E} \xi_{1}^{3}} \exp \left\{\frac{x^{3} g^{3}\left(t_{1}\right) \mathbb{E} \xi_{1}^{3}}{\left.6 n^{2} t_{1}^{2} \sigma^{4}\right\},}\right. & \text { если } \mathbb{E} \xi_{1}^{3}>0 \quad \text { и } \frac{x^{3}}{n^{2}} \rightarrow \infty, \\ \frac{12 n^{2} t_{2}^{3} \sigma^{4}}{x^{3} g^{3}\left(t_{2}\right)\left|\mathbb{E} \xi_{1}^{3}\right|} \exp \left\{\frac{x^{3} g^{3}\left(t_{2}\right) \mathbb{E} \xi_{1}^{3}}{6 n^{2} t_{2}^{2} \sigma^{4}}\right\}, & \text { если } \mathbb{E} \xi_{1}^{3}<0 \quad \text { и } \frac{x^{3}}{n^{2}} \rightarrow \infty .\end{cases}
$$

\section{§2. Доказательство теоремы 1}

Без ограничения общности всюду в дальнейшем считаем, что $\sigma=1$ и $g\left(t_{0}\right)=\sqrt{t_{0}}$. В противном случае мы можем провести следующую перенормировку:

$$
\mathbb{P}\left\{S_{k}>x g(k / n)\right\}=\mathbb{P}\left\{\frac{S_{k}}{\sigma}>\frac{x g(k / n)}{\sigma}\right\}=\mathbb{P}\left\{\frac{S_{k}}{\sigma}>\frac{x g\left(t_{0}\right)}{\sigma \sqrt{t_{0}}} \frac{\sqrt{t_{0}} g(k / n)}{g\left(t_{0}\right)}\right\}
$$


что немедленно сведет нашу задачу к указанному выше случаю при замене $x$ и $g$ соответственно на

$$
\widehat{x}:=\frac{x g\left(t_{0}\right)}{\sigma \sqrt{t_{0}}} \text { и } \widehat{g}(\cdot):=\frac{\sqrt{t_{0}} g(\cdot)}{g\left(t_{0}\right)} .
$$

Также без ограничения общности можно считать, что функция $g(t)$ равномерно ограничена. В противном случае часть суммы, где $g(k / n) \geq$ $2 g\left(t_{0}\right)$, можно мажорировать величиной $\mathbb{E} \tau_{n}\left(2 g\left(t_{0}\right) x\right)$, исследованной, например, в [1], которая не будет влиять на асимптотику $\mathbb{E} \tau_{n}(x g)$ (см. нижеследующую оценку (23)).

В силу замечания 1 мы рассмотрим только случай $m \geq 2$. Прежде всего выберем последовательность положительных чисел $\varepsilon_{1, n} \rightarrow 0$ таким образом, чтобы выполнялись следующие соотношения:

$$
\frac{n}{\varepsilon_{1, n} x^{2}} \rightarrow 0, \quad \frac{x}{\varepsilon_{1, n} n} \rightarrow 0
$$

Покажем, что такая последовательность $\varepsilon_{1, n}$ может быть выбрана в виде $\varepsilon_{1, n}=\delta_{n} \beta_{n}^{2 / m_{1}-2}$, где $\delta_{n} \rightarrow 0$. Проверка первого соотношения элементарна:

$$
\frac{n}{\varepsilon_{1, n} x^{2}}=\frac{1}{\varepsilon_{1, n} \beta_{n}^{2}}=\frac{1}{\delta_{n} \beta_{n}^{2 / m_{1}}} \rightarrow 0,
$$

если, например, $\delta_{n} \geq \beta_{n}^{-1 / m_{1}}$. Заметим, что из условия 3 следует асимптотическое представление $\beta_{n}=\nu_{n} n^{1 / 2-\gamma_{m}}$, где $\nu_{n} \rightarrow 0$, откуда получаем

$$
\frac{x}{\varepsilon_{1, n} n}=\frac{\beta_{n}}{\varepsilon_{1, n} \sqrt{n}}=\frac{\beta_{n}^{3-2 / m_{1}}}{\delta_{n} \sqrt{n}} \leq \frac{\beta_{n}^{3-2 / m}}{\delta_{n} \sqrt{n}}=\frac{\nu_{n}^{3-2 / m} n^{\left(1 / 2-\gamma_{m}\right)(3-2 / m)}}{\delta_{n} \sqrt{n}}=\frac{\nu_{n}^{3-2 / m}}{\delta_{n}} \rightarrow 0,
$$

скажем, если $\delta_{n} \geq \nu_{n}$. Таким образом, при $\delta_{n} \geq \max \left\{\beta_{n}^{-1 / m_{1}}, \nu_{n}\right\}$ и $\delta_{n} \rightarrow 0$ оба соотношения будут выполнены. В процессе доказательства теоремы 1 будет приведено еще одно условие на скорость убывания $\delta_{n}$.

Аналогичным образом введем последовательность $\varepsilon_{2, n}$, удовлетворяющую условиям

$$
\frac{n}{\varepsilon_{2, n} x^{2}} \rightarrow 0, \quad \frac{x}{\varepsilon_{2, n} n} \rightarrow 0 .
$$

Теперь разобьем $\mathbb{E} \tau_{n}(x g)$ на четыре подсуммы:

где

$$
\mathbb{E} \tau_{n}(x g)=R_{1}+R_{2}+R_{3}+R_{4},
$$

$$
\begin{array}{rlrl}
R_{1}:= & \sum_{k=N_{n}}^{\left[n_{0}\right]} \mathbb{P}\left\{S_{k}>x g(k / n)\right\}, & R_{2}:=\sum_{k=\left[n_{0}\right]+1}^{K_{n}} \mathbb{P}\left\{S_{k}>x g(k / n)\right\}, \\
R_{3}:=\sum_{k=1}^{N_{n}-1} \mathbb{P}\left\{S_{k}>x g(k / n)\right\}, & R_{4}:=\sum_{k=K_{n}+1}^{n} \mathbb{P}\left\{S_{k}>x g(k / n)\right\},
\end{array}
$$




$$
N_{n}:=\left[n t_{0}-\frac{n^{2} t_{0}^{2}}{\varepsilon_{1, n} x^{2}}\right], \quad K_{n}:=\left[n t_{0}+\frac{n^{2} t_{0}^{2}}{\varepsilon_{2, n} x^{2}}\right], \quad n_{0}:=n t_{0} .
$$

Если же $t_{0}=1$, то $R_{2}=R_{4}=0$. Далее будет показано, что $R_{3}=o\left(R_{1}\right)$ и $R_{4}=o\left(R_{2}\right)$, т. е. основной вклад в асимптотику $\mathbb{E} \tau_{n}(x g)$ вносят $R_{1}$ и $R_{2}$. Всюду далее будем полагать, что $t_{0} \in(0,1)$. В противном случае нам достаточно найти лишь асимптотику суммы $R_{1}$ и доказать, что $R_{3}=o\left(R_{1}\right)$. Это является частным случаем теоремы при $t_{0}=1$.

2.1. Исследование асимптотики $R_{1}+R_{2}$. Доказательство теоремы будет приводиться при $m_{1}>1$ и $m_{2}>1$. Если одно из этих двух чисел будет равно 1, то к соответствующей сумме можно применить рассуждения из [1].

Найдем асимптотику суммы $R_{1}$. Выводы при нахождении асимптотики $R_{2}$ будут зеркальными отражениями формул при вычислении асимптотики $R_{1}$, о чем более подробно будет сказано в конце параграфа.

Нам понадобится следующий результат из [1], непосредственно вытекающий из соответствующего утверждения в [3, с. 322].

Следствие. При выполнении условий 3 и 4 при $n \rightarrow \infty$ справедливо асимптотическое представление

$$
\mathbb{P}\left\{S_{n} \geq x\right\} \sim \frac{\sqrt{n} e^{-n \Lambda(x / n)}}{x \sqrt{2 \pi}}
$$

В силу условия 4 функция $\Lambda(z)$ аналитична в окрестности нуля. Прежде всего отметим, что из условия 3 равномерно по всем $k \in\left[N_{n}, n_{0}\right]$ имеют место предельные соотношения $x / k^{3 / 4} \rightarrow 0$ и $x / \sqrt{k} \rightarrow \infty$ при $n \rightarrow \infty$. Тогда при выполнении условий 1-4 нормированная функция уклонений при разложении в ряд Тейлора в окрестности нуля (ряд Крамера) примет вид

$$
k \Lambda\left(\frac{x g(k / n)}{k}\right)=\frac{x^{2} g^{2}(k / n)}{2 k}-\frac{\mathbb{E} \xi_{1}^{3} x^{3} g^{3}(k / n)}{6 k^{2}}+o(1) .
$$

Докажем, что для всех $k \in\left[N_{n}, n_{0}\right]$ выполняется следующая эквивалентность:

$$
\exp \left\{\frac{\mathbb{E} \xi_{1}^{3} x^{3} g^{3}(k / n)}{6 k^{2}}\right\} \sim \exp \left\{\frac{\mathbb{E} \xi_{1}^{3} x^{3} g^{3}\left(t_{0}\right)}{6 n^{2} t_{0}^{2}}\right\}=\exp \left\{\frac{\mathbb{E} \xi_{1}^{3} x^{3}}{6 n^{2} \sqrt{t_{0}}}\right\}
$$

или, что то же самое,

$$
\frac{x^{3} g^{3}(k / n)}{k^{2}}-\frac{x^{3}}{n^{2} \sqrt{t_{0}}}=\left(\frac{x^{3} g^{3}(k / n)}{k^{2}}-\frac{x^{3} g^{3}\left(t_{0}\right)}{k^{2}}\right)+\left(\frac{x^{3} g^{3}\left(t_{0}\right)}{k^{2}}-\frac{x^{3}}{n^{2} \sqrt{t_{0}}}\right) \rightarrow 0 .
$$


Покажем, что каждая из двух разностей в (5) будет стремиться к 0. Из условия 2 следует, что производная функции $G(t)$ равномерно ограничена в некоторой окрестности точки $t_{0}$, а следовательно,

$$
\left|g^{\prime}(t)\right|<C_{1}<\infty \text { для всех } t \in\left[\frac{N_{n}}{n}, \frac{n_{0}}{n}\right] .
$$

Из формулы конечных приращений следует, что

$$
g\left(\frac{k}{n}\right)-\sqrt{t_{0}}=g\left(\frac{k}{n}\right)-g\left(\frac{n_{0}}{n}\right) \leq C_{1} \frac{n_{0}-k}{n} \leq C_{1} \frac{t_{0} n_{0}}{\varepsilon_{1, n} x^{2}} .
$$

Воспользуемся неравенством

$$
\left|y^{3}-x^{3}\right| \leq 3 \max \left\{y^{2}, x^{2}\right\}|y-x|
$$

и оценкой $g(t)<2 \sqrt{t_{0}}$ для всех $t \in(0,1]$. Тогда

$$
\left|\frac{x^{3} g^{3}(k / n)}{k^{2}}-\frac{x^{3} g^{3}\left(t_{0}\right)}{k^{2}}\right| \leq 12 t_{0} \frac{x^{3}}{k^{2}}\left|g\left(\frac{k}{n}\right)-g\left(t_{0}\right)\right| \leq \frac{12 t_{0}^{2} C_{1} x^{3} n_{0}}{k^{2} \varepsilon_{1, n} x^{2}}=C_{2} \frac{x n_{0}}{k^{2} \varepsilon_{1, n}} .
$$

Из условия на $k$ следует, что $n_{0}-k \leq n_{0}^{2} / x^{2} \varepsilon_{1, n}$. Стало быть,

$$
C_{2} \frac{x n_{0}}{k^{2} \varepsilon_{1, n}} \leq C_{2} \frac{x n_{0}}{\left(n_{0}-n_{0}^{2} / x^{2} \varepsilon_{1, n}\right)^{2} \varepsilon_{1, n}}=C_{2} \frac{x}{n_{0} \varepsilon_{1, n}\left(1-n_{0} / x^{2} \varepsilon_{1, n}\right)^{2}} \rightarrow 0,
$$

так как $x / n_{0} \varepsilon_{1, n} \rightarrow 0$ и $n_{0} / x^{2} \varepsilon_{1, n} \rightarrow 0$ в силу условий на $\varepsilon_{1, n}$.

Рассмотрим вторую разность из (5). Для нее имеем

$$
\begin{aligned}
\left|\frac{x^{3} g^{3}\left(t_{0}\right)}{k^{2}}-\frac{x^{3}}{n^{2} \sqrt{t_{0}}}\right| & \leq x^{3} t_{0}^{3 / 2}\left(\frac{1}{\left(n_{0}-n_{0}^{2} / x^{2} \varepsilon_{1, n}\right)^{2}}-\frac{1}{n_{0}^{2}}\right) \\
& =\frac{x^{3} t_{0}^{3 / 2}}{n_{0}^{4}}\left(\frac{2 n_{0} / x^{2} \varepsilon_{1, n}-n_{0}^{2} / x^{4} \varepsilon_{1, n}^{2}}{\left(1-n_{0} / x^{2} \varepsilon_{1, n}\right)^{2}}\right) \rightarrow 0,
\end{aligned}
$$

здесь мы воспользовались тем, что $x^{3} / n_{0}^{4} \rightarrow 0$ по условию 3.

Таким образом, соотношение (5) доказано. Отсюда можно сделать вывод, что основной вклад в асимптотику показателя экспоненты вносит первый член его разложения в ряд Крамера. Поэтому в силу (3)-(5) верно следующее асимптотическое представление вероятности $\mathbb{P}\left\{S_{k}>x g(k / n)\right\}$ равномерно по всем $k \in\left[N_{n}, n_{0}\right]$ :

$$
\begin{aligned}
\mathbb{P}\left\{S_{k}>x g(k / n)\right\} & \sim \frac{\sqrt{k}}{x g\left(t_{n k}\right) \sqrt{2 \pi}} \exp \left\{-\frac{x^{2} g^{2}\left(t_{n k}\right)}{2 k}+\frac{\mathbb{E} \xi_{1}^{3} x^{3}}{6 n^{2} \sqrt{t_{0}}}\right\} \\
& =\frac{\sqrt{t_{n k}}}{\beta_{n} g\left(t_{n k}\right) \sqrt{2 \pi}} \exp \left\{-\frac{\beta_{n}^{2} g^{2}\left(t_{n k}\right)}{2 t_{n k}}+\frac{\mathbb{E} \xi_{1}^{3} x^{3}}{6 n^{2} \sqrt{t_{0}}}\right\},
\end{aligned}
$$


где $t_{n k}:=k / n$. Отсюда заключаем, что при $n \rightarrow \infty$

$$
R_{1} \sim n \exp \left\{\frac{\mathbb{E} \xi_{1}^{3} x^{3}}{6 n^{2} \sqrt{t_{0}}}\right\} \sum_{k=N_{n}}^{\left[n_{0}\right]} \frac{1}{n} \cdot \frac{\sqrt{t_{n k}}}{\beta_{n} g\left(t_{n k}\right) \sqrt{2 \pi}} \exp \left\{-\frac{\beta_{n}^{2} g^{2}\left(t_{n k}\right)}{2 t_{n k}}\right\} .
$$

Легко видеть, что выражение

$$
\frac{R_{1}}{n} \exp \left\{-\frac{\mathbb{E} \xi_{1}^{3} x^{3}}{6 n^{2} \sqrt{t_{0}}}\right\}
$$

эквивалентно интегральной сумме Римана соответствующего интеграла, которым и можно будет заменить правую часть (7), как будет установлено далее. Нам понадобится следующее утверждение, доказанное в [1].

Лемма 1. При $n \rightarrow \infty$ имеет место следующее соотношение:

$$
\begin{aligned}
& \left|\sum_{k=N_{n}}^{\left[n_{0}\right]} \frac{1}{n} \frac{\sqrt{t_{n k}}}{\beta_{n} g\left(t_{n k}\right) \sqrt{2 \pi}} \exp \left\{-\frac{\beta_{n}^{2} g^{2}\left(t_{n k}\right)}{2 t_{n k}}\right\}-\int_{N_{n} / n}^{t_{0}} \frac{\sqrt{t}}{g(t) \beta_{n} \sqrt{2 \pi}} \exp \left\{-\frac{\beta_{n}^{2} g^{2}(t)}{2 t}\right\} d t\right| \\
& \quad=o\left(\sum_{k=N_{n}}^{\left[n_{0}\right]} \frac{1}{n} \frac{\sqrt{t_{n k}}}{\beta_{n} g\left(t_{n k}\right) \sqrt{2 \pi}} \exp \left\{-\frac{\beta_{n}^{2} g^{2}\left(t_{n k}\right)}{2 t_{n k}}\right\}\right) .
\end{aligned}
$$

Воспользовавшись леммой 1, мы получили интеграл

$$
\int_{N_{n} / n}^{t_{0}} \frac{\sqrt{t}}{g(t)} e^{-\beta_{n}^{2} g^{2}(t) / 2 t} d t=\int_{N_{n} / n}^{t_{0}} \frac{1}{\sqrt{G(t)}} e^{-\beta_{n}^{2} G(t) / 2} d t
$$

Далее, заметим, что функция $G(t)$ непрерывна и монотонно убывает на полуинтервале $\left[N_{n} / n, t_{0}\right)$ по причине того, что при больших $n$ область интегрирования будет включаться в окрестность $\left[t_{0}-\delta, t_{0}\right]$ точки $t=t_{0}$. Функция $e^{-\beta_{n}^{2} G(t) / 2}$ на этом полуинтервале интегрируема, $G(t)=1+o(1)$ при $n \rightarrow \infty$ равномерно по всем $t \in\left[N_{n} / n, t_{0}\right)$. Поэтому к интегралу (8) применима теорема о среднем:

$$
\int_{N_{n} / n}^{t_{0}} \frac{1}{\sqrt{G(t)}} e^{-\beta_{n}^{2} G(t) / 2} d t \sim \int_{N_{n} / n}^{t_{0}} e^{-\beta_{n}^{2} G(t) / 2} d t .
$$

Разложим функцию $\mathrm{G}(\mathrm{t})$ в ряд Тейлора в окрестности точки $t=t_{0}-0$ :

$$
\begin{aligned}
G(t) & =\sum_{j=0}^{m_{1}} \frac{G^{(j)}\left(t_{0}-0\right)\left(t-t_{0}\right)^{j}}{j !}+\Delta\left(t-t_{0}\right)\left(t-t_{0}\right)^{m_{1}} \\
& =1+\frac{G^{\left(m_{1}\right)}\left(t_{0}-0\right)\left(t-t_{0}\right)^{m_{1}}}{m_{1} !}+\Delta\left(t-t_{0}\right)\left(t-t_{0}\right)^{m_{1}}
\end{aligned}
$$


где $\Delta(y)$ - некоторая функция, стремящаяся к 0 при $y \rightarrow 0$. Тогда интеграл в правой части (9) принимает следующий вид:

$$
\begin{aligned}
& \int_{N_{n} / n}^{t_{0}} e^{-\beta_{n}^{2} G(t) / 2} d t \\
& \quad=\int_{N_{n} / n}^{t_{0}} \exp \left\{-\frac{\beta_{n}^{2}}{2}\left(1+\frac{G^{\left(m_{1}\right)}\left(t_{0}-0\right)\left(t-t_{0}\right)^{m_{1}}}{m_{1} !}+\Delta\left(t-t_{0}\right)\left(t-t_{0}\right)^{m_{1}}\right)\right\} d t .
\end{aligned}
$$

Отметим, что длина интервала интегрирования $\left(t_{0}-N_{n} / n\right)$ в последнем интеграле имеет порядок

$$
\frac{n}{\varepsilon_{1, n} x^{2}}=\frac{1}{\delta_{n} \beta_{n}^{2 / m_{1}}}
$$

Без ограничения общности можно считать, что $\Delta(y)$ при $y \rightarrow+0$ монотонно убывает. Теперь оценим величину погрешности в показателе экспоненты под указанным интегралом:

$$
\beta_{n}^{2}\left(t_{0}-\frac{N_{n}}{n}\right)^{m_{1}} \Delta\left(t_{0}-\frac{N_{n}}{n}\right) \sim \frac{1}{\delta_{n}^{m_{1}}} \Delta\left(\frac{1}{\delta_{n} \beta_{n}^{2 / m_{1}}}\right) \rightarrow 0
$$

при достаточно медленно стремящейся к нулю последовательности $\delta_{n} \geq$ $\beta_{n}^{-1 / m_{1}}$. При такой оценке снизу эту последовательность можно выбрать с условием $\delta_{n} \geq \Delta^{1 /\left(m_{1}+1\right)}\left(\beta_{n}^{-1 / m_{1}}\right)$. Это и есть третье и последнее ограничение на скорость сходимости к нулю последовательности $\delta_{n}$. Например, последовательность

$$
\delta_{n}=\max \left\{\nu_{n}, \beta_{n}^{-1 / m_{1}}, \Delta^{1 /\left(m_{1}+1\right)}\left(\beta_{n}^{-1 / m_{1}}\right)\right\}
$$

удовлетворяет всем трем вышеприведенным условиям.

Таким образом, окончательно получаем

$$
\int_{N_{n} / n}^{t_{0}} e^{-\beta_{n}^{2} G(t) / 2} d t \sim e^{-\beta_{n}^{2} / 2} \int_{N_{n} / n}^{t_{0}} \exp \left\{-\frac{\beta_{n}^{2}}{2 m_{1} !} G^{\left(m_{1}\right)}\left(t_{0}-0\right)\left(t-t_{0}\right)^{m_{1}}\right\} d t .
$$

Проведем замену интегрирования по формуле $y=\left(t_{0}-t\right)^{m_{1}}$. Тогда интеграл в правой части последнего соотношения примет следующий вид:

$$
\begin{aligned}
& e^{-\beta_{n}^{2} / 2} \int_{N_{n} / n}^{t_{0}} \exp \left\{-\frac{\beta_{n}^{2}}{2 m_{1} !} G^{\left(m_{1}\right)}\left(t_{0}-0\right)\left(t-t_{0}\right)^{m_{1}}\right\} d t \\
& \quad=e^{-\beta_{n}^{2} / 2} \int_{0}^{\left(t_{0}-N_{n} / n\right)^{m_{1}}} \frac{1}{m_{1}} y^{1 / m_{1}-1} \exp \left\{-\frac{\beta_{n}^{2}}{2 m_{1} !}(-1)^{m_{1}} G^{\left(m_{1}\right)}\left(t_{0}-0\right) y\right\} d y .
\end{aligned}
$$

Функция $G(t)$ убывает в некоторой окрестности $t_{0}$ при $t<t_{0}$. Из формулы $(10)$ следует, что при нечетных $m_{1}$ выполняется $G^{\left(m_{1}\right)}\left(t_{0}-0\right)<0$, а 
при четных $m_{1}$ будет $G^{\left(m_{1}\right)}\left(t_{0}-0\right)>0$, т. е. величина $(-1)^{m_{1}} G^{\left(m_{1}\right)}\left(t_{0}-0\right)$ положительна. Таким образом,

$$
\begin{gathered}
e^{-\beta_{n}^{2} / 2} \int_{0}^{\left(t_{0}-N_{n} / n\right)^{m_{1}}} \frac{1}{m_{1}} y^{1 / m_{1}-1} \exp \left\{-\frac{\beta_{n}^{2}}{2 m_{1} !}(-1)^{m_{1}} G^{\left(m_{1}\right)}\left(t_{0}-0\right) y\right\} d y \\
\sim \frac{1}{m_{1}} B_{m_{1}}^{1 / m_{1}} e^{-\beta_{n}^{2} / 2} \int_{0}^{\left(t_{0}-N_{n} / n\right)^{m_{1}} B_{m_{1}}^{-1}} z^{1 / m_{1}-1} e^{-z} d z
\end{gathered}
$$

где

$$
B_{m_{1}}:=\frac{2 m_{1} !}{\beta_{n}^{2}\left|G^{\left(m_{1}\right)}\left(t_{0}-0\right)\right|} .
$$

Поведение верхнего предела интегрирования при $n \rightarrow \infty$ по существу уже установлено в (11):

$$
\left(t_{0}-\frac{N_{n}}{n}\right)^{m_{1}} B_{m_{1}}^{-1}=O\left(\delta_{n}^{-m_{1}}\right) .
$$

Из (13) следует, что верхний предел интеграла в правой части (12) стремится к $\infty$. Потому при $n \rightarrow \infty$ имеем

$$
\begin{aligned}
\frac{1}{m_{1}} B_{m_{1}}^{1 / m_{1}} e^{-\beta_{n}^{2} / 2} \int_{0}^{\left(t_{0}-N_{n} / n\right)^{m_{1}} B_{m_{1}}^{-1}} z^{1 / m_{1}-1} e^{-z} d z \\
\quad \sim \frac{1}{m_{1}} B_{m_{1}}^{1 / m_{1}} e^{-\beta_{n}^{2} / 2} \int_{0}^{\infty} z^{1 / m_{1}-1} e^{-z} d z \\
\quad=\frac{1}{m_{1}} B_{m_{1}}^{1 / m_{1}} e^{-\beta_{n}^{2} / 2} \Gamma\left(\frac{1}{m_{1}}\right)=B_{m_{1}}^{1 / m_{1}} e^{-\beta_{n}^{2} / 2} \Gamma\left(\frac{m_{1}+1}{m_{1}}\right)
\end{aligned}
$$

Из леммы 1, а также соотношений (6), (8) и (14) мы получаем следующую асимптотику:

$$
\begin{aligned}
R_{1} & \sim \frac{n B_{m_{1}}^{1 / m_{1}}}{\sqrt{2 \pi} \beta_{n}} \exp \left\{-\frac{x^{2}}{2 n}+\frac{\mathbb{E} \xi_{1}^{3} x^{3}}{6 n^{2} \sqrt{t_{0}}}\right\} \Gamma\left(\frac{m_{1}+1}{m_{1}}\right) \\
& \sim \frac{n\left(2 m_{1} !\right)^{1 / m_{1}}}{\sqrt{2 \pi} \beta_{n}^{1+2 / m_{1}}\left|G^{\left(m_{1}\right)}\left(t_{0}-0\right)\right|^{1 / m_{1}}} \exp \left\{-\frac{x^{2}}{2 n}+\frac{\mathbb{E} \xi_{1}^{3} x^{3}}{6 n^{2} \sqrt{t_{0}}}\right\} \Gamma\left(\frac{m_{1}+1}{m_{1}}\right) \\
& =\frac{n^{3 / 2+1 / m_{1}}\left(2 m_{1} !\right)^{1 / m_{1}}}{\sqrt{2 \pi} x^{1+2 / m_{1}}\left|G^{\left(m_{1}\right)}\left(t_{0}-0\right)\right|^{1 / m_{1}}} \exp \left\{-\frac{x^{2}}{2 n}+\frac{\mathbb{E} \xi_{1}^{3} x^{3}}{6 n^{2} \sqrt{t_{0}}}\right\} \Gamma\left(\frac{m_{1}+1}{m_{1}}\right) .
\end{aligned}
$$

Таким образом, мы получили асимптотику для суммы $R_{1}$.

Для вычисления асимптотики $R_{2}$ повторим всю схему рассуждений при нахождении асимптотики $R_{1}$. Первым делом обратимся к формуле $(5)$. 
Путем повторения рассуждений для $k \in\left[n_{0}, K_{n}\right]$ можно убедиться, что аналог формулы (5) также будет выполняться. Ясно, что аналог леммы 1 также применим и к

а именно:

$$
\frac{R_{2}}{n} \exp \left\{-\frac{\mathbb{E} \xi_{1}^{3} x^{3}}{6 n^{2} \sqrt{t_{0}}}\right\}
$$

$$
\frac{R_{2}}{n} \exp \left\{-\frac{\mathbb{E} \xi_{1}^{3} x^{3}}{6 n^{2} \sqrt{t_{0}}}\right\} \sim \int_{t_{0}}^{K_{n} / n} \frac{1}{\sqrt{G(t)}} \exp \left\{-\frac{\beta_{n}^{2} G(t)}{2}\right\} d t .
$$

В силу условий на функцию $G(t)$ теорема о среднем здесь также применима. Далее, с помощью аналогов формул (10) и (11) получаем

$$
\int_{t_{0}}^{K_{n} / n} e^{-\beta_{n}^{2} G(t) / 2} d t \sim e^{-\beta_{n}^{2} / 2} \int_{t_{0}}^{K_{n} / n} \exp \left\{-\frac{\beta_{n}^{2}}{2 m_{2} !} G^{\left(m_{2}\right)}\left(t_{0}+0\right)\left(t-t_{0}\right)^{m_{2}}\right\} d t .
$$

Заметим, что аналог формулы (9) позволяет утверждать, что в интеграле в правой части $(16)$ величина $G^{\left(m_{2}\right)}\left(t_{0}+0\right)$ положительна. Заменим переменную интегрирования по формуле $y=\left(t-t_{0}\right)^{m_{2}}$. После замены переменной получаем следующий интеграл:

$$
\begin{aligned}
& e^{-\beta_{n}^{2} / 2} \int_{t_{0}}^{K_{n} / n} \exp \left\{-\frac{\beta_{n}^{2}}{2 m_{2} !} G^{\left(m_{2}\right)}\left(t_{0}+0\right)\left(t-t_{0}\right)^{m_{2}}\right\} d t \\
& \quad=e^{-\beta_{n}^{2} / 2} \int_{0}^{\left(K_{n} / n-t_{0}\right)^{m_{2}}} \frac{1}{m_{2}} y^{1 / m_{2}-1} \exp \left\{-\frac{\beta_{n}^{2}}{2 m_{2} !} G^{\left(m_{2}\right)}\left(t_{0}+0\right) y\right\} d y .
\end{aligned}
$$

Осталось лишь заметить, что полученный интеграл отличается от интеграла в $(12)$ только аргументом у функции $G^{\left(m_{1}\right)}(\cdot)$ и соответственно константой $m_{2}$ вместо $m_{1}$. Верхний предел интегрирования аналогично формуле (13) будет стремиться к $\infty$. В итоге для $R_{2}$ имеем

$$
R_{2} \sim \frac{n^{3 / 2+1 / m_{2}}\left(2 m_{2} !\right)^{1 / m_{2}}}{\sqrt{2 \pi} x^{1+2 / m_{2}}\left|G^{\left(m_{2}\right)}\left(t_{0}+0\right)\right|^{1 / m_{2}}} \exp \left\{-\frac{x^{2}}{2 n}+\frac{\mathbb{E} \xi_{1}^{3} x^{3}}{6 n^{2} \sqrt{t_{0}}}\right\} \Gamma\left(\frac{m_{2}+1}{m_{2}}\right) .
$$

Сравним между собой асимптотики для $R_{1}$ и $R_{2}$, полученные в формулах (15) и (17). Утверждается следующее: если $m_{1}>m_{2}$, то $R_{2}=o\left(R_{1}\right)$. В самом деле, не обращая внимания на константы, которые не зависят от $n$, мы видим, что $R_{1}$ и $R_{2}$ отличаются множителями

$$
\frac{n^{3 / 2+1 / m_{1}}}{x^{1+2 / m_{1}}}=n \beta_{n}^{-1-2 / m_{1}}, \quad \frac{n^{3 / 2+1 / m_{2}}}{x^{1+2 / m_{2}}}=n \beta_{n}^{-1-2 / m_{2}} .
$$

Требуемое предельное соотношение будет выполняться, так как $\beta_{n} \rightarrow \infty$. Отсюда можно сделать вывод, что асимптотика будет определяться максимальным из двух номеров первых ненулевых производных. 
2.2. Оценка $R_{3}+R_{4}$. В этом пункте мы покажем, что $R_{3}+R_{4}$ не вносит вклада в асимптотику среднего времени, т. е. в (15) и (17) приведен ее окончательный вид.

Лемма 2. При $n \rightarrow \infty$ имеет место соотношение $R_{3}=o\left(R_{1}\right)$.

Доказательство. Разобьем сумму $R_{3}$ на две части: $R_{3}=R_{5}+R_{6}$, где

$$
R_{5}:=\sum_{k=b n t_{0}}^{N_{n}-1} \mathbb{P}\left\{S_{k}>g(k / n) x\right\}, \quad R_{6}:=\sum_{k=1}^{b n t_{0}-1} \mathbb{P}\left\{S_{k}>g(k / n) x\right\},
$$

а положительное $b<1$ будет выбрано позднее.

Сначала найдем асимптотику суммы $R_{5}$. Из (3) и (4) получается следующее представление для отношения вероятностей:

$$
\begin{aligned}
& \frac{\mathbb{P}\left(S_{k}>g(k / n) x\right)}{\mathbb{P}\left(S_{n t_{0}}>x \sqrt{t_{0}}\right)} \\
& \sim \frac{\sqrt{k}}{\sqrt{n} g(k / n)} \exp \left\{\frac{x^{2} g^{2}\left(t_{0}\right)}{2 n t_{0}}-\frac{x^{2} g^{2}(k / n)}{2 k}+\frac{\mathbb{E} \xi_{1}^{3} x^{3} g^{3}(k / n)}{6 k^{2}}-\frac{\mathbb{E} \xi_{1}^{3} x^{3} g^{3}\left(t_{0}\right)}{6 n^{2} t_{0}^{2}}\right\} .
\end{aligned}
$$

Из формулы конечных приращений и неравенства

$$
\left|y^{3}-x^{3}\right| \leq 3 \max \left\{y^{2}, x^{2}\right\}|y-x|
$$

вытекает следующая оценка для всех $k \in\left[b n t_{0}, N_{n}-1\right]$ :

$$
\begin{aligned}
& \left|\frac{\mathbb{E} \xi_{1}^{3} x^{3} g^{3}(k / n)}{6 k^{2}}-\frac{\mathbb{E} \xi_{1}^{3} x^{3} g^{3}\left(t_{0}\right)}{6 n^{2} t_{0}^{2}}\right| \\
& \quad \leq C_{1}\left|\frac{x^{3} g^{3}(k / n)}{k^{2}}-\frac{x^{3} g^{3}\left(t_{0}\right)}{k^{2}}\right|+C_{1}\left|\frac{x^{3} g^{3}\left(t_{0}\right)}{k^{2}}-\frac{x^{3} g^{3}\left(t_{0}\right)}{n^{2} t_{0}^{2}}\right| \\
& \quad \leq C_{1} \frac{x^{3}}{b^{2} n^{2} t_{0}^{2}} \max \left\{g^{2}(k / n), g^{2}\left(t_{0}\right)\right\}\left|g(k / n)-g\left(t_{0}\right)\right|+C_{1} x^{3} g^{3}\left(t_{0}\right) \frac{n^{2} t_{0}^{2}-k^{2}}{k^{2} n^{2} t_{0}^{2}} \\
& \quad \leq C_{2} \frac{x^{3}}{n^{2}}\left|k / n-t_{0}\right|+C_{1} x^{3} g^{3}\left(t_{0}\right) \frac{\left(n t_{0}-k\right)\left(n t_{0}+k\right)}{k^{2} n^{2} t_{0}^{2}} \\
& \quad \leq C_{2}(x / n)^{3}\left(n t_{0}-k\right)+C_{3} \frac{x^{3}}{n^{4}} 2 n t_{0}\left(n t_{0}-k\right) \leq C_{4}(x / n)^{3}\left(n t_{0}-k\right) .
\end{aligned}
$$

Мы без ограничения общности полагали, что функция $G(t)=g^{2}(t) / t$ в окрестности точки $t_{0}$ убывает слева и $G\left(t_{0}\right)=1$. Выберем достаточно малое число $\delta>0$, чтобы отрезок $\left[(1-\delta) n t_{0}, N_{n}\right]$ включался в такую окрестность. Так что для всех $k \in\left[(1-\delta) n t_{0}, N_{n}\right]$ получаем

$$
\begin{aligned}
\frac{g^{2}(k / n) x^{2}}{2 k}-\frac{x^{2}}{2 n} & =\frac{x^{2}}{2 n}(G(k / n)-1) \\
& =\frac{x^{2}}{2 n}\left(G(k / n)-G\left(t_{0}\right)\right) \geq \frac{x^{2}}{2 n^{2}}\left|G^{\prime}\left(\theta_{0}\right)\right|\left(n t_{0}-k\right) .
\end{aligned}
$$


Если же $k \in\left[b n t_{0},(1-\delta) n t_{0}\right]$ (при условии $\left.b<1-\delta\right)$, то в силу условия 1 имеем $G(k / n)>1+c(\delta)$, где $c(\delta)>0$, откуда вытекает оценка снизу

$$
\frac{g^{2}(k / n) x^{2}}{2 k}-\frac{x^{2}}{2 n}=\frac{x^{2}}{2 n}(G(k / n)-1) \geq c(\delta) \frac{x^{2}}{2 n^{2}} .
$$

Таким образом, из (19)-(21) и условия 3 следует равномерная по $k \in$ $\left[b n t_{0}, N_{n}\right]$ оценка сверху

$$
\left|\frac{x^{2} g^{2}\left(t_{0}\right)}{2 n t_{0}}-\frac{x^{2} g^{2}(k / n)}{2 k}+\frac{\mathbb{E} \xi_{1}^{3} x^{3} g^{3}(k / n)}{6 k^{2}}-\frac{\mathbb{E} \xi_{1}^{3} x^{3} g^{3}\left(t_{0}\right)}{6 n^{2} t_{0}^{2}}\right| \leq C_{5}\left(\frac{x}{n}\right)^{2}\left(n t_{0}-k\right) .
$$

Тогда в (18) для всех $k \in\left[b n t_{0}, N_{n}\right]$ при достаточно больших $n$ получаем

$$
\frac{\mathbb{P}\left(S_{k}>g(k / n) x\right)}{\mathbb{P}\left(S_{n t_{0}}>x \sqrt{t_{0}}\right)} \leq C_{6} \exp \left\{-C_{7}\left(\frac{x}{n}\right)^{2}\left(n t_{0}-k\right)\right\}
$$

где положительные постоянные $C_{k}$ в приведенных выше выкладках зависят только от функции $g$ и констант $b>0$ и $t_{0}$.

Таким образом, принимая во внимание (3), имеем

$$
\begin{aligned}
& \sum_{k=b n t_{0}}^{N_{n}-1} \mathbb{P}\left\{S_{k}>g(k / n) x\right\} \\
& \leq C_{6} \mathbb{P}\left(S_{n t_{0}}>x \sqrt{t_{0}}\right) \sum_{k=b n t_{0}}^{N_{n}} \exp \left\{-C_{7}\left(\frac{x}{n}\right)^{2}\left(n t_{0}-k\right)\right\} \\
& \quad \sim C_{6} \mathbb{P}\left(S_{n t_{0}}>x \sqrt{t_{0}}\right) \int_{b n t_{0}}^{N_{n}} \exp \left\{-C_{7} \frac{x^{2}}{n^{2}}\left(n t_{0}-t\right)\right\} d t \\
& =C_{6} \mathbb{P}\left(S_{n t_{0}}>x \sqrt{t_{0}}\right) \int_{n t_{0}-N_{n}}^{(1-b) n t_{0}} \exp \left\{-C_{7} \frac{x^{2}}{n^{2}} s\right\} d s \\
& =O\left(\mathbb{P}\left(S_{n t_{0}}>x \sqrt{t_{0}}\right) \frac{n^{2}}{x^{2}} \exp \left\{-C_{7} \frac{x^{2}}{n^{2}}\left(n t_{0}-N_{n}\right)\right\}\right) \\
& =O\left(\frac{n^{5 / 2}}{x^{3}} e^{-C_{7} t_{0}^{2} / \varepsilon_{1, n}} \exp \left\{-\frac{x^{2}}{2 n}+\frac{\mathbb{E}_{1}^{3} x^{3}}{6 n^{2} \sqrt{t_{0}}}\right\}\right)=o\left(R_{1}\right)
\end{aligned}
$$

Из оценок (15) и (22) следует утверждение леммы для $R_{5}$.

Нам осталось оценить $R_{6}$. Справедлива очевидная оценка

$$
R_{6} \leq \sum_{k=1}^{b n t_{0}} \mathbb{P}\left\{S_{k}>r x\right\} \equiv \mathbb{E} \tau_{\left[b n t_{0}\right]}(r x)
$$


Из [1] следует, что

$$
\mathbb{E} \tau_{\left[b n t_{0}\right]}(r x) \sim \frac{\sqrt{2}\left(b n t_{0}\right)^{5 / 2}}{\sqrt{\pi}(r x)^{3}} \exp \left\{-b n t_{0} \Lambda\left(\frac{r x}{\left(b n t_{0}\right)}\right)\right\}, \quad n \rightarrow \infty .
$$

Так как $r x /\left(b n t_{0}\right) \rightarrow 0$, в окрестности нуля можно использовать оценку для функции уклонений (см. (4))

$$
\Lambda\left(\frac{r x}{\left(b n t_{0}\right)}\right) \geq c \frac{(r x)^{2}}{2\left(b n t_{0}\right)^{2}}
$$

а это значит, что

$$
\exp \left\{-b n t_{0} \Lambda\left(\frac{r x}{\left(b n t_{0}\right)}\right)\right\} \leq \exp \left\{-c \frac{(r x)^{2}}{2 b n t_{0}}\right\} .
$$

Положим теперь $b=r^{2} /\left(2 t_{0}\right)$. Таким образом, для такого $b$ имеем

$$
R_{6}=o\left(R_{1}\right)
$$

что является завершающим шагом в доказательстве леммы.

Доказательство утверждения $R_{4}=o\left(R_{2}\right)$ полностью аналогично уже доказанному в лемме 2 . Таким образом, теорема 1 доказана.

\section{§3. Доказательство теоремы 2}

Мы так же, как и выше, будем полагать без ограничения общности, что функция $g(t)$ равномерно ограничена, $\sigma=1$ и $g(t)=\sqrt{t}$ для всех $t \in\left[t_{1}, t_{2}\right]$. Разобьем $\mathbb{E} \tau_{n}(x g)$ на три подсуммы: $\mathbb{E} \tau_{n}(x g)=S_{1}+S_{2}+S_{3}$, где

$$
\begin{gathered}
S_{1}:=\sum_{k=1}^{\left[n t_{1}\right]} \mathbb{P}\left\{S_{k}>x g(k / n)\right\}, \quad S_{2}:=\sum_{k=\left[n t_{1}\right]+1}^{\left[n t_{2}\right]} \mathbb{P}\left\{S_{k}>x g(k / n)\right\}, \\
S_{3}:=\sum_{k=\left[n t_{2}\right]+1}^{n} \mathbb{P}\left\{S_{k}>x g(k / n)\right\} .
\end{gathered}
$$

Всюду далее предполагается, что $t_{1} \neq 0$ и $t_{2} \neq 1$, в противном случае доказательство будет только упрощаться, так как хотя бы одна из двух сумм $S_{1}$ и $S_{3}$ будет равна 0 .

Поведение сумм $S_{1}$ и $S_{3}$ при $n \rightarrow \infty$ было изучено при доказательстве теоремы 1. Рассмотрим это на примере $S_{1}$. Применяя рассуждения при доказательстве теоремы 1 к сумме $S_{1}$, мы получаем для нее следующую асимптотику: 


$$
S_{1} \sim \frac{n^{3 / 2+1 / m_{1}}\left(2 m_{1} !\right)^{1 / m_{1}}}{\sqrt{2 \pi} x^{1+2 / m_{1}}\left|G^{\left(m_{1}\right)}\left(t_{1}-0\right)\right|^{1 / m_{1}}} \Gamma\left(\frac{m_{1}+1}{m_{1}}\right) \exp \left\{-n t_{1} \Lambda\left(\frac{x}{n \sqrt{t_{1}}}\right)\right\} .
$$

Для $S_{3}$ верна следующая асимптотика:

$$
S_{3} \sim \frac{n^{3 / 2+1 / m_{2}}\left(2 m_{2} !\right)^{1 / m_{2}}}{\sqrt{2 \pi} x^{1+2 / m_{2}}\left|G^{\left(m_{2}\right)}\left(t_{2}+0\right)\right|^{1 / m_{2}}} \Gamma\left(\frac{m_{2}+1}{m_{2}}\right) \exp \left\{-n t_{2} \Lambda\left(\frac{x}{n \sqrt{t_{2}}}\right)\right\} .
$$

Теперь перейдем к нахождению асимптотики суммы $S_{2}$. Заметим, что при данных условиях $g(k / n)=\sqrt{k / n}$ для всех $k \in\left[\left[n t_{1}\right]+1,\left[n t_{2}\right]\right]$. Применяя (3) к каждой вероятности, участвующей в сумме $S_{2}$, получаем, что для всех $k \in\left[\left[n t_{1}\right]+1,\left[n t_{2}\right]\right]$

$$
\begin{aligned}
\mathbb{P}\left\{S_{k}>x g(k / n)\right\} & \sim \frac{\sqrt{k}}{\sqrt{2 \pi} x g(k / n)} e^{-k \Lambda(x g(k / n) / k)} \\
& \sim \frac{\sqrt{n}}{\sqrt{2 \pi} x} \exp \left\{-\frac{x^{2}}{2 n}-k \Lambda_{0}\left(\frac{x}{\sqrt{k n}}\right)\right\} \\
& =\frac{\sqrt{n}}{\sqrt{2 \pi} x} \exp \left\{-\frac{x^{2}}{2 n}-n t_{n k} \Lambda_{0}\left(\frac{x}{n \sqrt{t_{n k}}}\right)\right\},
\end{aligned}
$$

где $t_{n k}:=k / n$. Отсюда следует представление для суммы $S_{2}$ :

$$
\begin{aligned}
S_{2} & =\sum_{k=\left[n t_{1}\right]+1}^{\left[n t_{2}\right]} \mathbb{P}\left\{S_{k}>x g(k / n)\right\} \\
& \sim \sum_{k=\left[n t_{1}\right]+1}^{\left[n t_{2}\right]} \frac{\sqrt{n}}{\sqrt{2 \pi} x} \exp \left\{-\frac{x^{2}}{2 n}-n t_{n k} \Lambda_{0}\left(\frac{x}{n \sqrt{t_{n k}}}\right)\right\} \\
& =\frac{n^{3 / 2}}{\sqrt{2 \pi} x} e^{-x^{2} / 2 n} \sum_{k=\left[n t_{1}\right]+1}^{\left[n t_{2}\right]} \frac{1}{n} \exp \left\{-n t_{n k} \Lambda_{0}\left(\frac{x}{n \sqrt{t_{n k}}}\right)\right\} .
\end{aligned}
$$

Заметим, что сумма, стоящая в конце формулы (27), представляет собой интегральную сумму Римана. Докажем следующий аналог леммы 1.

Лемма 3. При $n \rightarrow \infty$ имеет место следующее соотношение:

$$
\begin{gathered}
\left|\sum_{k=\left[n t_{1}\right]+1}^{\left[n t_{2}\right]} \frac{1}{n} \exp \left\{-n t_{n k} \Lambda_{0}\left(\frac{x}{n \sqrt{t_{n k}}}\right)\right\}-\int_{t_{1}}^{t_{2}} \exp \left\{-n t \Lambda_{0}\left(\frac{x}{n \sqrt{t}}\right)\right\} d t\right| \\
\quad=o\left(\sum_{k=\left[n t_{1}\right]+1}^{\left[n t_{2}\right]} \frac{1}{n} \exp \left\{-n t_{n k} \Lambda_{0}\left(\frac{x}{n \sqrt{t_{n k}}}\right)\right\}\right) .
\end{gathered}
$$


Доказательство. Известна элементарная оценка погрешности приближения интеграла Римана для любой функции $f \in C^{1}(0,1)$ :

$$
\left|\sum_{k=\left[n t_{1}\right]+1}^{\left[n t_{2}\right]} \frac{1}{n} f\left(t_{n k}\right)-\int_{t_{1}}^{t_{2}} f(t) d t\right| \leq \sum_{k=\left[n t_{1}\right]+1}^{\left[n t_{2}\right]} \frac{1}{n^{2}} \max _{t_{n, k-1} \leq t \leq t_{n k}}\left|f^{\prime}(t)\right| .
$$

Далее, полагая

$$
f(t)=\exp \left\{-n t \Lambda_{0}\left(\frac{x}{n \sqrt{t}}\right)\right\}
$$

покажем, что $k$-е слагаемое суммы в правой части последнего неравенства представляет собой бесконечно малую более высокого порядка, чем соответствующее слагаемое суммы в (27). Воспользовавшись представлением функции уклонений через ряд Крамера, получим

$$
f(t)=\exp \left\{-n t \Lambda_{0}\left(\frac{x}{n \sqrt{t}}\right)\right\}=\exp \left\{\frac{\mathbb{E} \xi_{1}^{3} x^{3}}{6 n^{2} \sqrt{t}}(1+o(1))\right\} .
$$

После вычисления производной $f^{\prime}(t)$ можно легко убедиться в том, что для доказательства леммы нам достаточно лишь заметить, что

$$
\frac{1}{n^{2}} \frac{\mathbb{E} \xi_{1}^{3} x^{3}}{12 n^{2} t^{3 / 2}}=O\left(\frac{x^{3}}{n^{4}}\right)=o\left(\frac{1}{n}\right)
$$

равномерно по всем $t$ из области интегрирования.

Нам осталось доказать, что $S_{1}=o\left(S_{2}\right)$ и $S_{3}=o\left(S_{2}\right)$, или, другими словами, показать, что именно $S_{2}$ определяет асимптотику всей суммы. Покажем это для $S_{1}=o\left(S_{2}\right)$. Доказательство $S_{3}=o\left(S_{2}\right)$ будет полностью аналогично приведенному ниже.

Прежде всего получим нижнюю оценку на $S_{2}$. Введем мажоранту $g_{2}(t)$ для функции $g(t)$ на интервале $\left(t_{1}, 1\right)$ таким образом, чтобы функция $G_{2}(t):=g_{2}^{2}(t) / t$ монотонно возрастала для всех $t \in\left(t_{1}, 1\right)$. Также потребуем, чтобы номер первой правой ненулевой производной функции $G_{2}(t)$ в точке $t_{1}$ был больше $m_{1}$ и был конечен. Обозначим его как $m_{3}$. Отсюда следует оценка

$$
S_{2}^{\prime}:=\sum_{k=\left[n t_{1}\right]+1}^{\left[n t_{2}\right]} \mathbb{P}\left\{S_{k}>x g_{2}(k / n)\right\} \leq S_{2}
$$

Осталось лишь заметить, что функция

$$
g_{1}(t):= \begin{cases}g(t), & t \in\left(0, t_{1}\right] \\ g_{2}(t), & t \in\left(t_{1}, 1\right)\end{cases}
$$


удовлетворяет условиям 1 и 2 , а потому с помощью теоремы 1 мы получаем следующую асимптотику:

$$
S_{2}^{\prime} \sim \frac{n^{3 / 2+1 / m_{3}}\left(2 m_{3} !\right)^{1 / m_{3}}}{\sqrt{2 \pi} x^{1+2 / m_{3}}\left|G^{\left(m_{3}\right)}\left(t_{1}+0\right)\right|^{1 / m_{3}}} \Gamma\left(\frac{m_{3}+1}{m_{3}}\right) \exp \left\{-n t_{1} \Lambda\left(\frac{x}{n \sqrt{t_{1}}}\right)\right\} .
$$

Сравнивая множители перед экспонентой, можно легко убедиться в том, что $S_{1}=o\left(S_{2}^{\prime}\right)$. Отсюда немедленно вытекает основное утверждение теоремы.

Далее уже отмечалось, что при $m>1$ мы имеем $x^{4} / n^{3} \rightarrow 0$. Тогда

$$
-n t \Lambda_{0}\left(\frac{x}{n \sqrt{t}}\right)=\frac{\alpha_{n}}{\sqrt{t}}+o(1), \quad \text { где } \quad \alpha_{n}:=\frac{\mathbb{E} \xi_{1}^{3} x^{3}}{6 n^{2}} .
$$

Найдем асимптотику интеграла $I_{n}:=\int_{t_{1}}^{t_{2}} e^{\alpha_{n} / \sqrt{t}} d t$. Рассмотрим 2 случая:

СлУЧАй 1. $\mathbb{E} \xi_{1}^{3}=0$ или $x^{3} / n^{2} \rightarrow 0$. Если $\mathbb{E} \xi_{1}^{3}=0$, то $\alpha_{n}=0$ для всех $n$. Поэтому $I_{n} \sim t_{2}-t_{1}$. При $x^{3} / n^{2} \rightarrow 0$ мы имеем $\alpha_{n} \rightarrow 0$, что снова дает нам $I_{n} \sim t_{2}-t_{1}$ по теореме Лебега.

СлУчАй 2. $x^{3} / n^{2} \rightarrow \infty$ и $\mathbb{E} \xi_{1}^{3} \neq 0$. Вычислим асимптотику интеграла $I_{n}$ :

$$
\begin{aligned}
I_{n} & =\int_{t_{1}}^{t_{2}} e^{\alpha_{n} / \sqrt{t}} d t=2 \alpha_{n}^{2} \int_{\alpha_{n} / \sqrt{t_{2}}}^{\alpha_{n} / \sqrt{t_{1}}} \frac{e^{y}}{y^{3}} d y \\
& =2 \alpha_{n}^{2}\left(\left.\frac{e^{y}}{y^{3}}\right|_{\alpha_{n} / \sqrt{t_{2}}} ^{\alpha_{n} / \sqrt{t_{1}}}+3 \int_{\alpha_{n} / \sqrt{t_{2}}}^{\alpha_{n} / \sqrt{t_{1}}} \frac{e^{y}}{y^{4}} d y\right) \\
& =2 \alpha_{n}^{2}\left(L_{n}+O\left(\frac{L_{n}}{\alpha_{n}}\right)\right) \\
& =2 \alpha_{n}^{2}\left(L_{n}+o\left(L_{n}\right)\right) \sim 2 \alpha_{n}^{2} L_{n}
\end{aligned}
$$

где

$$
L_{n}:=\left.\frac{e^{y}}{y^{3}}\right|_{\alpha_{n} / \sqrt{t_{2}}} ^{\alpha_{n} / \sqrt{t_{1}}}
$$

Из (28) получаем

$$
\begin{aligned}
I_{n} & \sim \frac{2 t_{1}^{3 / 2}}{\alpha_{n}} e^{-\alpha_{n} / \sqrt{t_{1}}}-\frac{2 t_{2}^{3 / 2}}{\alpha_{n}} e^{-\alpha_{n} / \sqrt{t_{2}}} \\
& =\frac{12 n^{2} t_{1}^{3 / 2}}{\mathbb{E} \xi_{1}^{3} x^{3}} \exp \left\{\frac{\mathbb{E} \xi_{1}^{3} x^{3}}{6 n^{2} \sqrt{t_{1}}}\right\}-\frac{12 n^{2} t_{2}^{3 / 2}}{\mathbb{E} \xi_{1}^{3} x^{3}} \exp \left\{\frac{\mathbb{E} \xi_{1}^{3} x^{3}}{6 n^{2} \sqrt{t_{2}}}\right\} .
\end{aligned}
$$


Нетрудно заметить, что

$$
I_{n} \sim \begin{cases}\frac{12 n^{2} t_{1}^{3 / 2}}{\mathbb{E} \xi_{1}^{3} x^{3}} \exp \left\{\frac{\mathbb{E} \xi_{1}^{3} x^{3}}{6 n^{2} \sqrt{t_{1}}}\right\}, & \text { если } \mathbb{E} \xi_{1}^{3}>0, \\ -\frac{12 n^{2} t_{2}^{3 / 2}}{\mathbb{E} \xi_{1}^{3} x^{3}} \exp \left\{\frac{\mathbb{E} \xi_{1}^{3} x^{3}}{6 n^{2} \sqrt{t_{2}}}\right\}, & \text { если } \mathbb{E} \xi_{1}^{3}<0 .\end{cases}
$$

Теорема 2 доказана.

Авторы благодарят А. А. Могульского за ряд полезных замечаний касательно постановки задачи в теореме 1.

\section{Список литературы}

1. Борисов И. С., Шефер Е. И. Асимптотика среднего времени пребывания траектории случайного блуждания выше удаляющейся криволинейной границы // Сиб. журн. чист. и прикл. матем. 2017. Т. 17, Вып. 4. С. 18-27.

2. Боровков A. А. Теория вероятностей. 5-е изд. / Учебное пособие. М.: Книжный дом «Либроком», 2009.

3. Боровков A. А., Боровков K. А. Асимптотический анализ случайных блужданий. Т. 1: Медленно убывающие распределения скачков. М.: Физматлит, 2008.

4. Лотов В. И., Тарасенко А. С. Об асимптотике среднего времени пребывания случайного блуждания на полуоси // Изв. РАН. Сер. матем. 2015. Т. 79, № 3. С. 23-40.

\section{Борисов Игорь Семенович}

Институт математики

им. С. Л. Соболева СО РАН,

просп. Академика Коптюга, 4,

Новосибирск, 630090 РОССИЯ.

Новосибирский гос. университет,

ул. Пирогова, 2,

Новосибирск, 630090 РОССИЯ.

E-mail: sibam@math.nsc.ru

\section{Шефер Евгений Игоревич}

Новосибирский гос. университет,

ул. Пирогова, 2 ,

Новосибирск, 630090 РОССИЯ.

E-mail: shef_john@mail.ru
Поступила в редакцию 30 января 2019 г.

Получена после доработки 14 февраля 2019 г.

Принята к публикации 27 февраля 2019 г. 